\title{
Review
}

\section{Muscle Cell Function and the Effects of Hyperbaric Oxygen Therapy}

\author{
Tammy Rossomando, MS.EHS, MEd, ATC* \\ Health and Safety Ergomedic-Consulting, Hillsdale, NJ 07642, USA \\ "Corresponding author \\ Tammy Rossomando, MS.EHS, MEd, ATC \\ President, Health and Safety Ergomedic-Consulting, Hillsdale, NJ 07642, USA;Tel. 20I-403-4I48; E-mail: tammyrossomando@gmail.com
}

\section{Article information}

Received: October $7^{\text {th }}, 2019$; Revised: Ocober 28 th $^{\text {t } 2019 ; ~ A c c e p t e d: ~ N o v e m b e r ~} 1^{\text {st }}, 2019$; Published: November $8^{\text {th }}, 2019$

\section{Cite this article}

Rossomando T. Muscle cell function and the effects of hyperbaric oxygen therapy. Orthop Res Traumatol Open J. 2019; 4(I): 6-9. doi: I0.17। 40/ORTOJ-4-II5

\section{ABSTRACT}

There are different processes via which a muscle cell can utilize oxygen to make energy that will sustain activity. The type of activity and duration of activity will determine what energy system is used to sustain the activity being done. Aerobic metabolism uses oxygen to sustain the energy demand. Oxygen is obtained from the air we breathe, and then transported to the cells though the myoglobin. Although ambient air only contains $21 \%$ oxygen, it is enough to sustain life and energy needs. But what if the muscle cell could instead receive 100\% oxygen? The delivery method would be via hyperbaric oxygen therapy (HBO) which supplies oxygen at $100 \%$ concentration under a minimum of 1 atmospheric pressure. Atmospheric pressure will affect the outcomes of consuming $100 \%$ oxygen. Research supports positive findings on oxygen therapy under pressure and muscle cell recovery but much research still needs to be investigated. One research study revealed detrimental effects of $100 \%$ oxygen breathed in consistently for days resulting in comorbidities that did not exist prior to experiment. HBO can pose some risks but if it is applied in a controlled manner, $\mathrm{HBO}$ can be a safe option to enhance cellular recovery and cell function.

\section{Keywords}

Oxygen; Hyperbaric oxygen therapy; Muscle recovery; Muscle cells; Pure oxygen.

\section{MUSCLE CELL FUNCTION ANDTHE EFFECTS OF HYPERBARIC OXYGENTHERAPY}

$\mathrm{T}$ The ambient air we breathe in contains only $21 \%$ oxygen; the air is not 'just' oxygen. It contains typically $78 \%$ nitrogen, $21 \%$ oxygen and the $1 \%$ remaining is a blend of carbon, helium, argon and hydrogen. As an interesting fact, the $1 \%$ are gasses that surround the earth and the closer to sea level we are breathing, the higher the percentage of oxygen. Other factors affect ambient air as well such as the pollution level. In a general statement, what we do as humans: manufacturing processes, transportation and chemicals we use, etc., affect the quality of the air we breathe. What if instead of decreasing air quality with our modernization efforts, we increase our breathing air quality by increasing the purity and concentration of oxygen we take in? What would the effect of taking in 100\% oxygen do? Would muscles work better, heal faster? After all, is not more better? ${ }^{1}$

\section{HOW MUSCLE CELLS WORK}

Muscle fiber contractions depend on excitation-contraction (EC) coupling and occur in a sequence of events. EC coupling in its simplistic form is an action potential that triggers a myocyte to contract, followed by subsequent relaxation. This is where the muscle cell filaments slide along each other, expanding and contracting the cell. The sarcoplasmic reticulum (SR) is a membrane structure found not only within muscle cells but it also resembles SR in other cells where its main function is to store the calcium ions needed for muscle cell functions including but not limited to manufacturing secreted proteins, integrating proteins in the cell membrane and facilitating glycosylation. The calcium ions regulate muscle contractions by allowing them to occur. The SR is a noteworthy structure in that it contains large amounts of myoglobin (a protein that assists in oxygen-binding and is found in red blood cells). This makes the blood the ideal oxygen transport system to the cells once the oxygen supply has been replenished from respiration. ${ }^{2}$

\section{PURPOSE OF OXYGEN}

The purpose of oxygen in the human body lies within each cell. Oxygen is used by each cell to assist in the process of converting stored energy that is stored in food to a useable form, referred to 
as cellular respiration, through a reaction with glucose. The purpose of this process is to power the cells, produce involuntary movements and move materials into and out of cells. ${ }^{3}$ The body has three major phases of cellular respiration: glycolysis (removes the hydrogen atoms by redox), the citric acid cycle (also known as the Krebs Cycle) and the electron transport system (ETS). The processes are always occurring as the body cells typically have a short life span and constantly degrade and rebuild an essential process for all biochemical reactions in the body. The human body has unending energy needs due to cellular metabolism being an ongoing process. ${ }^{4}$

\section{GLYCOLYSIS AND CELLULAR RESPIRATION}

The first process that occurs in cellular respiration is referred to as glycolysis and although this stage of anaerobic respiration can occur without oxygen, the process cannot continue without the assistance of oxygen. Oxygen is a vital component of human life. Lack of oxygen or insufficient amounts will initially impair function of the cells and eventually lead to cellular death followed by death of the host if adequate levels are not restored to functional capacity in a timely manner. Using oxygen for cellular respiration is referred to as glycolysis or aerobic respiration (meaning "with air"). The body uses oxygen and combines it with stored energy to convert it to a useable form by combining with glucose. Respiration without the use of oxygen is referred to as anaerobic respiration (meaning "without air"). How glycolysis gets produced will be very different depending on whether or not oxygen was used and if there was enough available to sustain the energy conversion process. If there was enough oxygen available to complete glycolysis at the time it was needed, the end product will be pyruvate. Pyruvate is a transport molecule that is needed to carry carbon atoms into the mitochondria to be oxidized to carbon dioxide and water (cellular waste). It then becomes part of the citric acid cycle (which occurs in the mitochondria of eukaryotic cells) and the process aerobically metabolizes carbohydrates, fats and proteins. This is where the cellular waste gets produced. The waste products of the process are the conversion of adenosine diphosphate (ADP) to adenosine triphosphate (ATP). ATP is the biochemical energy carrier for all living cells to store and use energy. If there was not enough oxygen to make energy readily available to sustain an activity level and the body's processes are requiring the energy, the end product will be lactate. $^{5}$

Let's look at a functional application of how this applies in the muscle cell. When a runner is sprinting a very short distance, initially the muscle cells will use the stored ATP and movement is sustained from this energy source for about 3-seconds. The next phase begins where molecules are broken down and rebuilt for quick energy. This whole energy conversion process occurs very quickly and can support the body's activity for about 8-10 seconds. Should the demand for energy continue based on continual need for oxygen to sustain energy production, the next sequential muscle cell energy acquisition comes from the anaerobic process and produces lactic acid produced from ATP made from glucose, derived from cellular glycogen. ATP here is made at a much slower rate than the previous system. With energy needs still in high demand, by the two-minute mark of sustained motion, oxygen is added to the ATP production process and this allows glucose to be completely broken down into carbon dioxide and water (waste products of aerobic energy synthesis). This is aerobic respiration. The aerobic system produces ATP the slowest but can sustain activity for the longest amount of time.

We have shown how the body uses and needs oxygen. As discussed prior, the ambient air we breathe is made up of only $21 \%$ oxygen. Wouldn't more be better? After all, with more oxygen surely the efficiency of the muscle cell will increase, effectively enabling us to sustain an improved activity level or even recover better through increased cellular function? The answer isn't that simple.

In a study that examined guinea pigs breathing 100\% oxygen at normal pressure for 48-hours, the results revealed that damage had occurred via fluid accumulation in the lungs and to the epithelial cells lining the alveoli as well as damage occurring to the pulmonary capillaries. ${ }^{6}$ Studies reveals that humans breathing $100 \%$ oxygen at normal pressure will experience:

- Fluid in the lungs

- Reduced rate of gas flow across the alveoli resulting in an increased effort to breathe to get enough oxygen

- Chest pains with deep breathing

- Total volume of exchangeable air in the lungs decreases by $17 \%$

- Mucus plugs local areas of collapsed alveoli (atelectasis)

From the results from this study, we see that consuming $100 \%$ oxygen has very detrimental effects on the body.

\section{APPLICATION OF IOO PERCENT OXYGEN}

Other factors come in to play as well with increased oxygen concentration consumption such as atmospheric pressure. The effects of breathing $100 \%$ oxygen change when atmospheric pressure changes. The study mentioned above looked at $100 \%$ oxygen consumed at 'normal' pressure (undefined in the study). We breathe at about 1 atmospheric pressure (determined at sea level). Air has weight. One atmospheric pressure is equivalent to 14.7 pounds per square inch (psi). ${ }^{6}$ This is the amount of pressure that is exerted on the body by the earth. Atmospheric pressure affects the lungs ability to breathe. For the lungs to be inflated, the pressure on the outside of the lungs must be greater than the pressure inside the lungs, this effectively fits the definition of diffusion. Then as we breathe, diffusion 'pushes' air into the lungs and also helps push oxygen across the membrane of the cells in the lungs. ${ }^{7,8}$ There is an inverse relationship of altitude to atmospheric pressure; atmospheric pressure will decease as altitude increases. Breathing becomes more difficult the higher you go. In the body, at high altitudes, air molecules are spread farther apart due to the decreased ambient air pressure, making it harder for people to breathe from the lack of sufficient oxygen available to enter the lungs. As indicated on the web resource at Craig Hospital, ${ }^{7}$ in Denver at an altitude of 5,280 feet, we breathe in $17 \%$ less oxygen than someone does at sea level and by 18,000 feet we breathe in $50 \%$ less oxygen. In the opposite 
direction, the deeper we go away from sea level, as in under the water, the pressure increases. Even at just a few feet below the water's surface, it becomes difficult to breath as the water pressure becomes too great for the breathing muscles to expand and contract. ${ }^{6}$ Research has been established that the body can only withstand up to 3 to 4 atmospheres of pressure and there is also a minimum. We actually need some atmospheric pressure on the body for it to function properly ( 0.47 millibars of atmospheric pressure is the minimum, referred to as the Armstrong limit, as per the physician that discovered this, Dr H. Armstrong) and that the body's oxygen pressure at about $21 \%$ is enough to saturate hemoglobin. The point here is that atmospheric pressure effects the body's ability to manage oxygen which is an essential element for cell function.

If oxygen is administered under pressure (greater than ambient air), injuries may occur such as acute oxygen poisoning with symptoms of nausea, dizziness, muscle twitches, blurred vision and seizures/convulsions. ${ }^{9}$ Consider diving as an example where divers breathe in atmospheric air at $21 \%$ oxygen under pressure from a tank while submersed at depth in water. The deeper the dive, the more pressure is placed on the lungs. We already know there is an inverse relationship of elevation to oxygen at atmospheric pressure but what about exposure to $100 \%$ oxygen under pressure at 3 times atmospheric pressure during controlled intervals such as in $\mathrm{HBO}$ (for about 1-2-hours) with infrequent applications of the treatment?

\section{HYPERBARIC OXYGENTHERAPY}

$\mathrm{HBO}$ therapy involves an outpatient treatment where the patient breathes $100 \%$ oxygen in a pressurized room or chamber at about three times normal atmospheric pressure. This enhances the body's capability to gather more oxygen. Research has shown that with controlled applications for specific conditions, this is an effective treatment including decompression sickness, serious infections, bubbles in the blood vessels and wounds that won't heal (from diabetes or a radiation injury). ${ }^{10}$

The difference between $\mathrm{HBO}$ causing extensive internal body damage and being therapeutic is when $100 \%$ oxygen is administered in a controlled environment under pressure. Another difference in causation between having detrimental or therapeutic outcomes is the length of time subjects were exposed to the $100 \%$ oxygen. In the studies using $100 \%$ oxygen over $\mathrm{x}$ amount of days was held as the constant variable in the studies. It is not logical or feasible to breathe $100 \%$ oxygen all day and every day at ambient atmospheric pressure, which is what Brain's ${ }^{4}$ study on guinea pigs has shown to have the most extensive damage from use. Was all the resultant tissue damage from the $100 \%$ oxygen at atmospheric pressure in his study or was it from the constant extended use of taking in $100 \%$ oxygen, or a combination? This is an example of how the application of HBO therapy can have very different outcomes depending on the application and conditions applied to.

Therapeutic outcomes result from increased oxygen being carried through the blood which is made possible through the administration in an increased atmosphere/pressure climate with- in a defined schedule. The benefits can help to fight bacteria and stimulate the release of growth factors and stem cells which both promote healing. ${ }^{10}$ Injured tissue requires an increased amount of oxygen to survive and HBO increases the amount of oxygen your blood can carry. When the increased oxygen reaches the cells, it helps restore blood gases and tissue function. This is what enhances the ability to fight infection and promote healing. Conditions that can be healed and have been identified with the use of $\mathrm{HBO}$ include:

- Anemia, severe

- Brain abscess

- Bubbles of air in your blood vessels (arterial gas embolism)

- Burn

- Decompression sickness

- Carbon monoxide poisoning

- Crushing injury

- Deafness, sudden

- Gangrene

- Infection of skin or bone that causes tissue death

- Nonhealing wounds, such as a diabetic foot ulcers

- Radiation injury

- Skin graft or skin flap at risk of tissue death

- Vision loss, sudden and painless

\section{EFFECTS OF LUNG OXYGEN UPTAKE ON MUSCLE}

\section{CELLS}

Regarding metabolic properties of muscle cells with reference to sports, the contractile muscle and energy turnover/frequency during exercise varies at different speeds depending on the rate of eccentric contractions. In a study conducted by Gaesser \& Brooks, 1975; Coast \&Welch, 1985, ${ }^{11}$ results demonstrated that pulmonary oxygen uptake does have a direct positive correlation to increased exercise rate. What remained unknown was whether the increased pulmonary oxygen uptake also effected the oxygen uptake of contracting muscles (and therefore muscle cells). To further explore the effect of enhanced oxygen availability on muscle cells, let's consider how it can be transported by blood in two ways: chemically, bound to hemoglobin, and physically, dissolved in plasma. ${ }^{9}$ Oxygen binds to Hemoglobin by binding to an iron atom and then the oxygen saturated hemoglobin molecules carry the oxygen to the cells and carbon dioxide to the lungs. In a normal breathing environment, the body's normal hemoglobin has an oxygen saturation rate averaging around $97 \%$. Consequently, during HBO, hemoglobin is also fully saturated on the venous side, and the result is an increased oxygen tension throughout the vascular bed. Since diffusion is driven by a difference in tension, oxygen will be forced further out into tissues from the vascular bed. ${ }^{12}$ The short-term effects on the muscle cells from HBO therapy include vasoconstriction and enhanced oxygen delivery, reduction of edema, phagocytosis activation and an anti-inflammatory effect. ${ }^{13}$ On the longer-term side, the benefits include neovascularization, osteogenesis and stimulation of collagen production by fibroblasts (great for wound healing and recovery from radiation injury). ${ }^{12}$ Theoretically, as shown by research here, the answer to the researchers question that went unanswered in their study as to whether the increased pulmonary oxygen up- 
take also effected the oxygen uptake of contracting muscles (and thus muscle cells), the answer would be yes.

In studies conducted on humans where muscle soreness was purposefully induced and HBO therapy was strategically administered. Control groups were used in randomized, controlled, double-blind studies, the gold standard for research. The results from several studies all concluded that hyperbaric oxygen has an inhibitory effect on the inflammatory process, there was a significant recovery of eccentric torque but they also agreed despite this accelerated healing and there was no decrease in pain/discomfort from the delayed onset of muscle soreness (DOMS). In a similar study design to the above, it revealed a stimulation of aerobic oxidation to the mitochondria. This conclusion was supported by a similar study that revealed lactic acid and ammonia are removed more rapidly with the $\mathrm{HBO}$ treatment leading to a faster recovery time.

During the Nagano Winter Olympics, scientists used $\mathrm{HBO}$ as a recovery method for muscle fatigue. Athletes received HBO treatment for only 30-40-minutes (note the short duration time) and received up to a maximum of six treatments. The outcome showed that all athletes benefitted from the treatment as evidenced through faster recovery times.

\section{CONCLUSION}

Muscle cell metabolism has been shown to be enhanced by HBO. In some studies, HBO therapy lead to faster recovery times and supports oxygen saturation during hemoglobin transport to cells. Despite the positive cellular effects demonstrated through the research, a few studies had participants remain in discomfort with the effects of DOMS. This begs to question and encourage more research on enhanced positive cellular activity with improved muscle cell function and the negative effects of cellular breakdown and restructuring. Delivering 100\% pure oxygen under 2-3 atmospheric pressure on a therapeutic schedule has shown positive results and with this application, injuries have been drastically reduced. Some further research studying the applications of the therapeutic administration would benefit this topic's next steps. Perhaps with varying daily frequency or application time, other conditions can be abetted. What about doing very light exercise while getting $\mathrm{HBO}$ 'treatment', what effect would that have?

\section{REFERENCES}

1. Babul S, Rhodes EC. The role of hyperbaric oxygen therapy in sports medicine. Sports Med. 2000; 30(6): 395-403. doi: 10.2165/00007256-200030060-00002
2. ATP: What is it and why is it important? Web site. https://www. bodybuilding.com/content/atp-what-is-it-and-why-is-it-important.html. Accessed October 6, 2019.

3. Ori J. What do our body cells do with oxygen? Web site. https:// sciencing.com/do-body-cells-do-oxygen-6388828.html. Accessed October 6, 2019.

4. Lamb GD, Westerblad H. Acute effects of oxygen and nitrogen species on the contractile function of skeletal muscle. The Journal of Physiology. 2011; 589: 2119-2127. doi: 10.1113/jphysiol.2010.199059

5. Staughton J. What is Glycolysis? Web site. https://www.scienceabc.com/pure-sciences/what-is-glycolysis.html. Accessed October 6, 2019 .

6. Brain M. Is it harmful to breathe 100-percent oxygen? Web site. https://science.howstuffworks.com/question493.htm. Accessed October 6, 2019.

7. CRAIG Hospital. How We Breathe. Web site. https://craighospital.org/resources/how-we-breathe. Accessed October 6, 2019.

8. Gibb N. Under Pressure-Scuba Diving Risks, the Basic consequences of Depth and Pressure. Web site. https://www.liveabout. com/depth-and-pressure-scuba-diving-2963200. Accessed October 6, 2019.

9. Barata P, Cervaens M, Resende R, Camacho O, Marques F. Hyperbaric oxygen effects on sports injuries. Ther Adv Musculoskelet Dis. 2013; 3(2): 111-121. doi: 10.1177/1759720X11399172

10. Mayo Clinic. Hyperbaric Oxygen Therapy. https://www.mayoclinic.org/tests-procedures/hyperbaric-oxygen-therapy/about/ pac-20394380. Accessed October 6, 2019.

11. Ferguson RA, Ball D, Krustrup P, et al. Muscle oxygen uptake and energy turnover during dynamic exercise at different contraction frequencies in humans. J Physiol. 2001; 536(Pt 1): 261-271. doi: 10.1111/j.1469-7793.2001.00261.x

12. Mayer R, Hamilton-Farrell MR, van der Kleij AJ, et al. Hyperbaric oxygen and radiotherapy. Strablenther Onkol. 2005; 181(2): 113-123. doi: 10.1007/s00066-005-1277-y

13. Mortensen CR. Hyperbaric oxygen therapy. Current Anesthesia Critical Care. 2008; 19: 333-337. doi: 10.1016/j.cacc.2008.07.007 